\title{
A multi-technique approach for characterizing the SVN49 signal anomaly, part 2: chip shape analysis
}

\author{
Steffen Thoelert • Michael Meurer • \\ Stefan Erker • Oliver Montenbruck • \\ André Hauschild · Pat Fenton
}

Received: 9 November 2010/Accepted: 8 January 2011/Published online: 4 February 2011

(C) The Author(s) 2011. This article is published with open access at Springerlink.com

\begin{abstract}
Due to a satellite internal reflection at the L5 test payload, the SVN49 (PRN1) GPS satellite exhibits a static multipath on the L1 and L2 signals, which results in elevation-dependent tracking errors for terrestrial receivers. Using a 30-m high-gain antenna, code and carrier phase measurements as well as raw in-phase and quadrature radio frequency samples have been collected during a series of zenith passes in mid-April 2010 to characterize the SVN49 multipath and its impact on common users. Following an analysis of the receiver tracking data and the IQ constellation provided in Part 1 of this study, the present Part 2 provides an in-depth investigation into chip shapes for the L1 and L2 signals. A single reflection model is found to be compatible with the observed chip shape distortions and key parameters for an elevation dependent multipath model are derived. A good agreement is found between multipath parameters derived independently from raw IQ-samples and measurements of a so-called Vision Correlator. The chip shapes and their observed variation with elevation can be
\end{abstract}

Disclaimer: The choice of GNSS receivers and other test equipment employed for the SVN49 signal analysis is based on availability at the time of the test. Neither DLR nor the GPS Wing endorse or recommend any specific commercial product.

S. Thoelert · M. Meurer $\cdot$ S. Erker

Institute of Communications and Navigation,

Deutsches Zentrum für Luft- und Raumfahrt,

82230 Wessling, Germany

O. Montenbruck $(\bowtie) \cdot$ A. Hauschild

German Space Operations Center, Deutsches Zentrum

für Luft- und Raumfahrt, 82230 Wessling, Germany

e-mail: oliver.montenbruck@dlr.de

P. Fenton

NovAtel Inc, Calgary, AB, Canada used to predict the multipath response of different correlator types within a tracking receiver. The multipath model itself is suitable for implementation in a signal simulator and thus enables laboratory testing of actual receiver hardware.

Keywords Multipath, SVN49 - PRN1 - IQ sampling · Impulse response - Chip shape $\cdot$ Vision Correlator

\section{Introduction}

The near-zenith passage of the SVN49 GPS satellite over Germany in mid April 2010 offered a unique opportunity to study the satellite internal multipath on the L1 and L2 signals over the full range of boresight and elevation angles. In cooperation with the GPS Wing, a dedicated test campaign was conducted with the $30 \mathrm{~m}$ deep space antenna of DLR's German Space Operations Center (GSOC) at Weilheim. The high gain $(52 \mathrm{~dB})$ and narrow beam of this antenna enables an excellent signal-to-noise ratio and minimizes the impact of terrestrial multipath that might affect measurements with a standard GPS antenna. For a comprehensive characterization of the SVN49 internal multipath, a set of four multifrequency tracking receivers was operated in parallel to two spectrum analyzers for recording the digitized radio-frequency $(\mathrm{R} / \mathrm{F})$ samples at high sampling rates.

In the first part of this study (Hauschild et al. 2011; henceforth referred to as Part 1), reference profiles of the tracking errors for the civil (C/A, L2C) and precise $\mathrm{P}(\mathrm{Y})$ signals on the L1 and L2 frequency as well as the analysis of the in-phase/quadrature (IQ) constellation diagram have been presented. Based on the ionosphere- and geometry-free multipath combination, pseudorange errors of $-0.2 \mathrm{~m}$ (low elevation) to $+1.7 \mathrm{~m}$ (near zenith) are derived for $\mathrm{L} 1 \mathrm{C} / \mathrm{A}$ code receivers using conventional 
1 chip early-late correlator. A marginally lower amplitude of the multipath errors $(-0.15 \mathrm{~m}$ to $1.5 \mathrm{~m})$ is obtained for narrow correlator with a 0.1 chip spacing as well as $\mathrm{P}(\mathrm{Y})$ code tracking. For L2 in contrast, the pseudorange errors exhibit a notably smaller variation of $-0.4 \mathrm{~m}$ to $+0.2 \mathrm{~m}$ with its minimum near $60^{\circ}$ elevation. For an initial characterization of the reflected signal causing these errors, the observed histogram of IQ samples has been decomposed into a direct and a reflected component. Similar to the analysis of the receiver-tracking data, the impact of the reflected component in the IQ diagram is assumed to vanish near elevations of $40^{\circ}$ and $30^{\circ}$ for L1 and L2, respectively, which correspond to a steep minimum in the gain pattern of the antenna diagram for the $\mathrm{J} 2$ input port of the coupler network (Lake and Stansell 2009).

This contribution focuses on the observed chip shapes and the retrieval of amplitude, delay, and phase shift of the reflected signal based on a single-reflection multipath model. The employed method is essentially free of a priori assumptions on the antenna gain pattern and can be applied to lower relative power levels of the reflected signal than the analysis of the IQ histogram. The observed chip shapes can, furthermore, be used to synthesize the tracking errors that would be obtained with common correlator designs, and a good match with actual receiver tracking results is obtained. Finally, we compare the chip shapes derived from the high-rate raw data sampling with those obtained by the Vision Correlator of one of the tracking receivers. While restricted to the $\mathrm{L} 1 \mathrm{C} / \mathrm{A}$ code signal, the Vision Correlator can provide chip shape measurements using a standard low-gain antenna and is not limited to short snap-shot data takes as the raw sampling of the $\mathrm{R} / \mathrm{F}$ signals.

\section{Data sets and preprocessing}

For an in-depth analysis of the SVN49 signal distortions, high-rate $\mathrm{R} / \mathrm{F}$ samples were collected during the nearzenith passes over the Weilheim ground station from April 8 to 19 . Using an Agilent E4443 spectrum analyzer connected to the L-band feed of the 30-m antenna (Thölert et al. 2009), data recordings at different sampling rates, durations, and frequencies were performed in a fully automated and remotely controlled process. Typically, $100 \mathrm{~ms}$ data takes were obtained once every $100 \mathrm{~s}$ throughout each $7 \mathrm{~h}$ pass, while longer $1 \mathrm{~s}$ data takes were conducted at 15-min intervals.

At a $102.4 \mathrm{MHz}$ data collection rate, measurements from the in-phase (I) and quadrature (Q) channel of the spectrum analyzer are provided approximately once every $10 \mathrm{~ns}$, which offers a resolution of about 100 samples per C/A code chip and 10 samples per $\mathrm{P}(\mathrm{Y})$ code chip. The available bandwidth of $80 \mathrm{MHz}$ well exceeds the satellite's internal bandwidth limitation and thus enables a distortionfree monitoring of the transmitted signal.

Since the raw IQ samples are obtained after mixing with the nominal L1 or L2 reference frequency, a Doppler correction is performed in the post-processing. Here, a software-based phase-locked loop (PLL) is used to align the chip transitions with the nominal orientation in the IQ plane. In view of the high antenna gain, a representative signal-to-noise amplitude ratio of $20: 1$ (or $13 \mathrm{~dB}$ ) is achieved for the individual $10 \mathrm{~ns}$ IQ samples, and the modulation from the various signal components stands out clearly from the system noise.

As described in Part 1, various geodetic receivers were operated at the $30-\mathrm{m}$ antenna in parallel to the signals analyzer, in order to assess the impact of the SVN49 multipath on the tracking performance. Among these, the NovAtel OEMV receiver offers a so-called Vision Correlator, which aims to eliminate multipath-related tracking errors through comparison of measured chip shapes with a reference obtained in multipath-free conditions (Fenton and Jones 2005). For this purpose, the Vision Correlator performs in-phase and quadrature correlations of the L1 $\mathrm{C} / \mathrm{A}$ code signal on a grid of 110 bins covering a full $\mathrm{C} / \mathrm{A}$ code chip with an approximate spacing. Compared to raw sampling with a spectrum analyzer described above, the Vision Correlator operates as part of the normal receiver tracking and is able to deliver chip shape measurements on a continuous basis and without dedicated post-processing. On the other hand, it involves an additional bandwidth limitation of the receiver and is limited to the L1 C/A code signal. The individual 1 s samples exhibit a typical noise of $2 \%$ of the signal amplitude and is thus of a similar order of magnitude as the chip shape distortions to be investigated. The measurements have, therefore, been averaged over $1^{\circ}$ elevation bins, corresponding to intervals of 120-170 s duration, which reduces the noise by more than a factor of ten and facilitates the analysis of chip shape variations as a function of elevation or boresight angle.

\section{Impulse response and chip shape extraction}

The actual signal of a GNSS may be considered as the results of various forms of distortions applied to an ideal signal with purely rectangular chip shapes throughout the entire transmission chain. These distortions will typically include a bandwidth limitation but may also comprise multipath due to superposition of the direct and a reflected signal. Knowing the chip sequence $\underline{s}(t)$ of the ideal modulation, the transmitted signal $\underline{s}_{\mathrm{TX}}(t)$ may be written as a convolution 
$\underline{s}_{\mathrm{TX}}(t)=\underline{s}(t) * \underline{h}(t)$

of the ideal signal with the impulse response function (IRF) $\underline{h}(t)$. Here and in the sequel, underbars are used to designate complex quantities. The impulse response is typically time- and band-limited and can be represented by the time discrete approximation

$\underline{h}(t)=\sum_{\mu} \underline{h}_{\mu} \cdot \operatorname{sinc}\left(\frac{t-\mu T_{s}}{T_{s}}\right)$

which is defined through a limited number of samples $\underline{h}_{\mu}$ at equidistant steps at a sampling interval $T_{s}$. When sampling the observed and ideal signals on the same interval, the convolution (1) can be replaced by a matrix equation and an optimum estimate of the IRF for a given transmission system can be determined from the solution of a linear least squares problem (Meurer et al. 2010).

In practice, the transmitted signal of a GNSS satellite can be measured with a high-gain antenna and suitable R/F sampling equipment, but the ideal signal itself is not known in full detail beforehand. For the Block IIR-M satellite generation, a total of three modulations (C/A-code, $\mathrm{P} / \mathrm{Y}$ code, and M-code) are jointly transmitted on the L1 frequency along with an inter-modulation product (IM) on the quadrature channel that ensures a constant power envelope. The resulting signal may thus be written as

$$
\begin{aligned}
\underline{s}(t)= & \left(a_{1} \cdot c_{\mathrm{P}(\mathrm{Y})} \cdot s_{\mathrm{P}(\mathrm{Y})}+a_{2} \cdot c_{\mathrm{M}} \cdot s_{\mathrm{M}}\right) \\
& +j \cdot\left(a_{3} \cdot c_{\mathrm{C} / \mathrm{A}} \cdot s_{\mathrm{C} / \mathrm{A}}-a_{4} \cdot c_{\mathrm{IM}} \cdot s_{\mathrm{IM}}\right)
\end{aligned}
$$

were $s_{k}$ with $k \in\{\mathrm{C} / \mathrm{A}, \mathrm{P}(\mathrm{Y}), \mathrm{M}, \mathrm{IM}\}$ denotes the elementary shapes of a single chip (including, where applicable, the corresponding subcarrier modulation), $c_{k}$ is the sequence of code elements, and $a_{i}$ is the relative amplitude of the respective signal component. For the L2 frequency, a similar signal structure applies with L2C used instead of the civil C/A code. While the basic modulations, i.e., binary phase shift key BPSK(1) or binary offset carrier $\operatorname{BOC}(10,5)$, and fundamental periods of each of these signals are known, the actual code sequences are only available for the unencrypted C/A and L2C signals. However, the favorable signal-to-noise ratio obtained with the high-gain antenna enables a "read-out" of the actual code sequence from the sequence of $\mathrm{R} / \mathrm{F}$ samples. For best reliability, a matched filtering approach is employed in this process (Whalen 1971), which takes into account the known chip shapes $s_{i}$ and their timing relations. Even though the M-code signal and the P-code encryption were deactivated for some of the SVN49 passes over the Weilheim antenna to facilitate the raw data analysis, the method presented here is general enough to cope with multiple signal components and classified ranging codes. It is thus widely applicable to all other GNSS satellites in orbit right now or planned to be launched in the foreseeable future.
Having identified the code sequences for a given data set, the amplitudes $a_{i}$ remain as unknowns and prevent a full specification of the nominal signal $\underline{s}(t)$. To overcome this limitation, the vector $\left(h_{\mu}\right)_{\forall \mu}$ of discretized IRF samples is augmented with the four unknowns $\left(a_{1}, \ldots, a_{4}\right)$ and both parameter sets are jointly estimated from the observed total signal $\underline{s X}_{\mathrm{TX}}(t)$ as well as the four individual signal sequences $c_{k}(t) \cdot s_{k}(t)$ in a combined least-squares adjustment by minimizing the loss function

$J=\left\|\underline{s}_{\mathrm{TX}}(t)-\underline{s}\left(t ; a_{1}, \ldots, a_{4}\right) * \underline{h}\left(t ; h_{\mu}\right)\right\|$

over the entire set of sampling times $\left(t_{v}\right)$. For the purpose of illustration, Table 1 provides a summary of the estimated power ratios for the individual SVN49 signals, while Fig. 1 (left) shows the estimated IRF for an L1 signal observed at high elevation.

Upon convolution of the ideal chip shapes with the IRF, the observed chip shapes for the C/A (or L2C), P(Y) and M codes as well as the inter-modulation product are obtained (Meurer et al. 2010). The recovered chip shapes show a substantial "ringing" for all modulations, which results from the combined effect of a band limitation of approximately $\pm 20 \mathrm{MHz}$ as well as the superimposed multipath. For example, Fig. 2 shows the estimated chip shapes for the three L1 signal codes as well as the inter-modulation product, while Fig. 3 provides the P-Code chip shapes on L1 and L2 for three selected elevations. All results are based on the analysis of 20-ms data samples and have been normalized to nominal chip states of 0 (low) and +1 (high) and unit power over time. In case of the L1 signal, the impact of bandwidth limitation and multipath can readily be discerned from a comparison of the chip shape observed near zenith (i.e., with a maximum multipath amplitude) and the respective results for a data set obtained at $40^{\circ}$ elevation (where the reflected signal is substantially suppressed). It is also evident that a notable leakage between I and Q channels occurs in all signals near the various chip transitions.

The impulse response function corresponding to the above examples is shown in Fig. 4. Note that the IRF does not refer to a specific modulation but is likewise applicable for the C/A, $\mathrm{P}(\mathrm{Y})$, and $\mathrm{M}$ codes. The IRF exhibits a maximum near $40 \mathrm{~ns}$ ( $0.04 \mathrm{C} / \mathrm{A}$ code chips), which reflects the aforementioned bandwidth limitation of the transmitter.

Table 1 Power of the SVN49 signal components relative to the $\mathrm{P}(\mathrm{Y})$ code of the respective frequency (excluding possible signal distortions due to band limitation)

\begin{tabular}{llllll}
\hline Frequency & $\mathrm{C} / \mathrm{A}$ & $\mathrm{L} 2 \mathrm{C}(\mathrm{L}+\mathrm{M})$ & $\mathrm{P}(\mathrm{Y})$ & $\mathrm{M}$ & $\mathrm{IM}$ \\
\hline $\mathrm{L} 1$ & $+2.4 \mathrm{~dB}$ & $\mathrm{n} / \mathrm{a}$ & $0 \mathrm{~dB}$ & $+2.7 \mathrm{~dB}$ & $+0.4 \mathrm{~dB}$ \\
$\mathrm{~L} 2$ & $\mathrm{n} / \mathrm{a}$ & $-0.2 \mathrm{~dB}$ & $0 \mathrm{~dB}$ & $+0.0 \mathrm{~dB}$ & $+0.4 \mathrm{~dB}$ \\
\hline
\end{tabular}



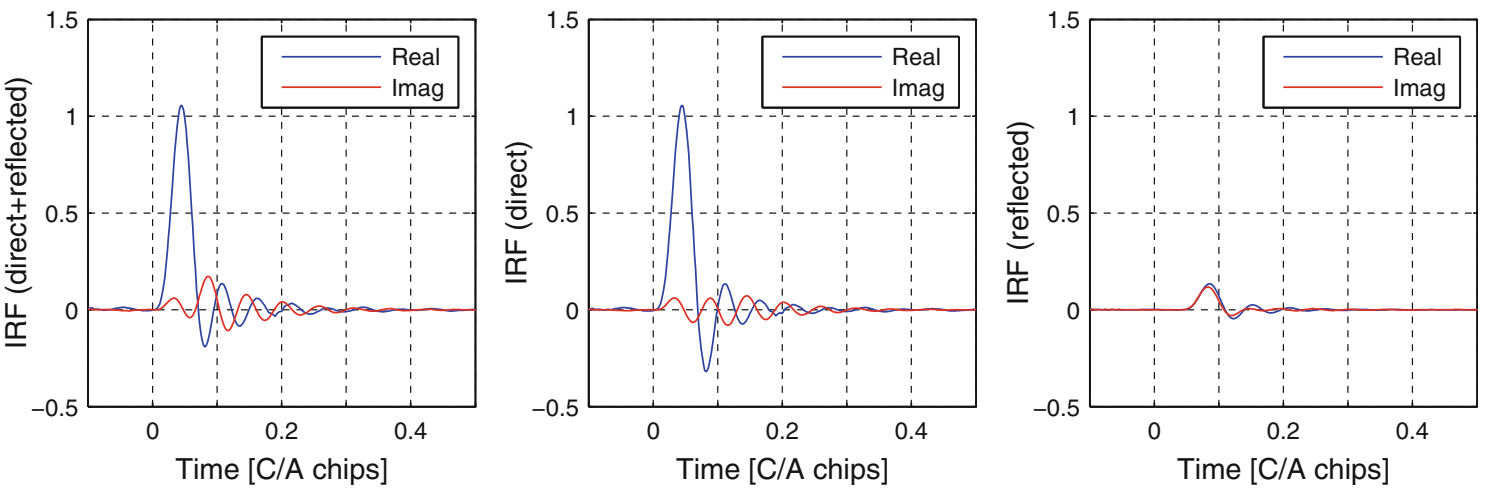

Fig. 1 Separation of direct and reflected signal contribution of the impulse response function. Combined IRF $\underline{h}(t)$ (left), direct signal IRF $\underline{h}_{0}(t)$ (center) and reflected contribution $\underline{\alpha}_{0} \underline{h}_{0}(t-\tau)($ right $)$ for $90^{\circ}$ elevation angle
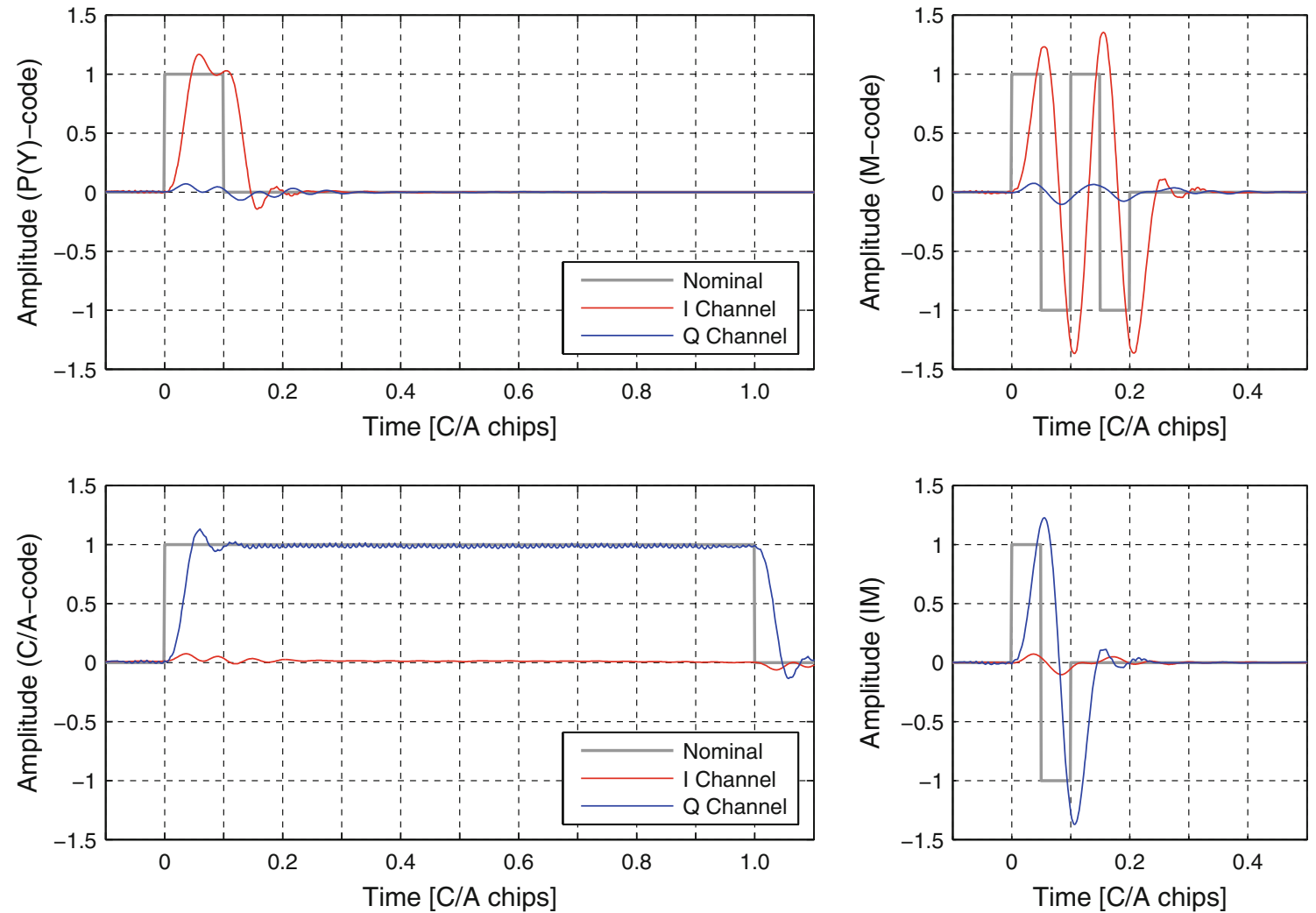

Fig. 2 Estimated chip shapes of $\mathrm{P}(\mathrm{Y})$-code, M-code, C/A-code, and inter-modulation product (from top left to bottom right) for the GPS SVN49 L1 signal at $50^{\circ}$ elevation on 22 April 2010

Substantial amplitude of the imaginary part of the IRF (roughly $10 \%$ of the real valued part) can, furthermore, be recognized, which reflects the observed mixing between I and $\mathrm{Q}$ channels near chip transitions.

\section{Multipath parameter estimation}

Having derived the impulse response, or equivalently, the associated chip shapes of the transmission chain, the contributions from the direct and reflected signals need to be separated. As discussed by Weill (2002), the decomposition can be achieved by fitting a reference chip shape for the direct signal as well as a delayed and phase shifted copy to the observed chip shape. For given delays of the direct and reflected signal components relative to the reference chip shape, the amplitude and phases of both signal components can be estimated in a linear least-squares approach, which leaves a two-dimensional nonlinear minimization problem for the determination of the delay values. 

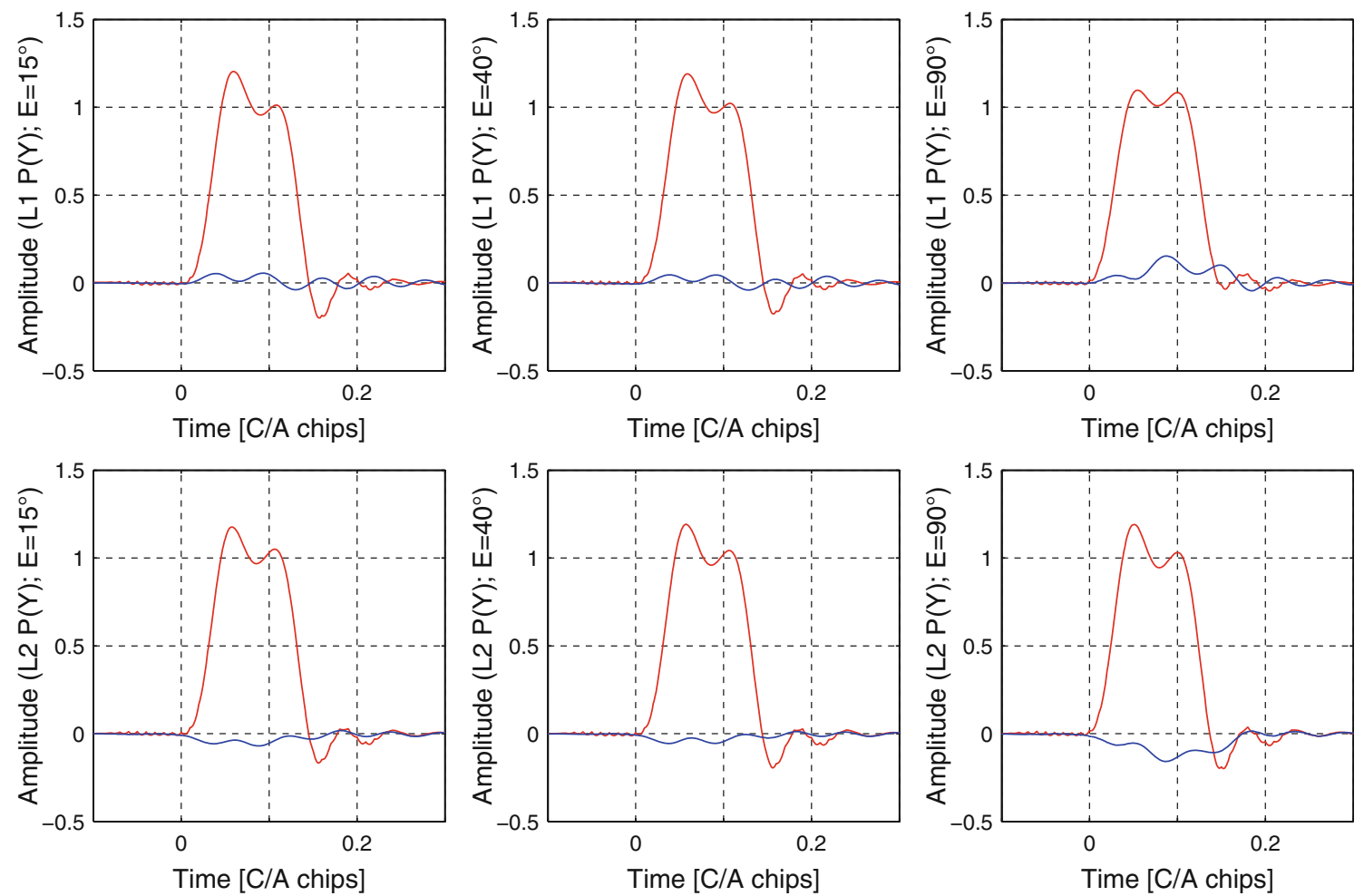

Fig. 3 P-code chip shapes for L1 and L2 signal at $15^{\circ}$ (left), $40^{\circ}$ (center) and $90^{\circ}$ (right) elevation on 16 April 2010 (red: I channel, blue: Q channel)
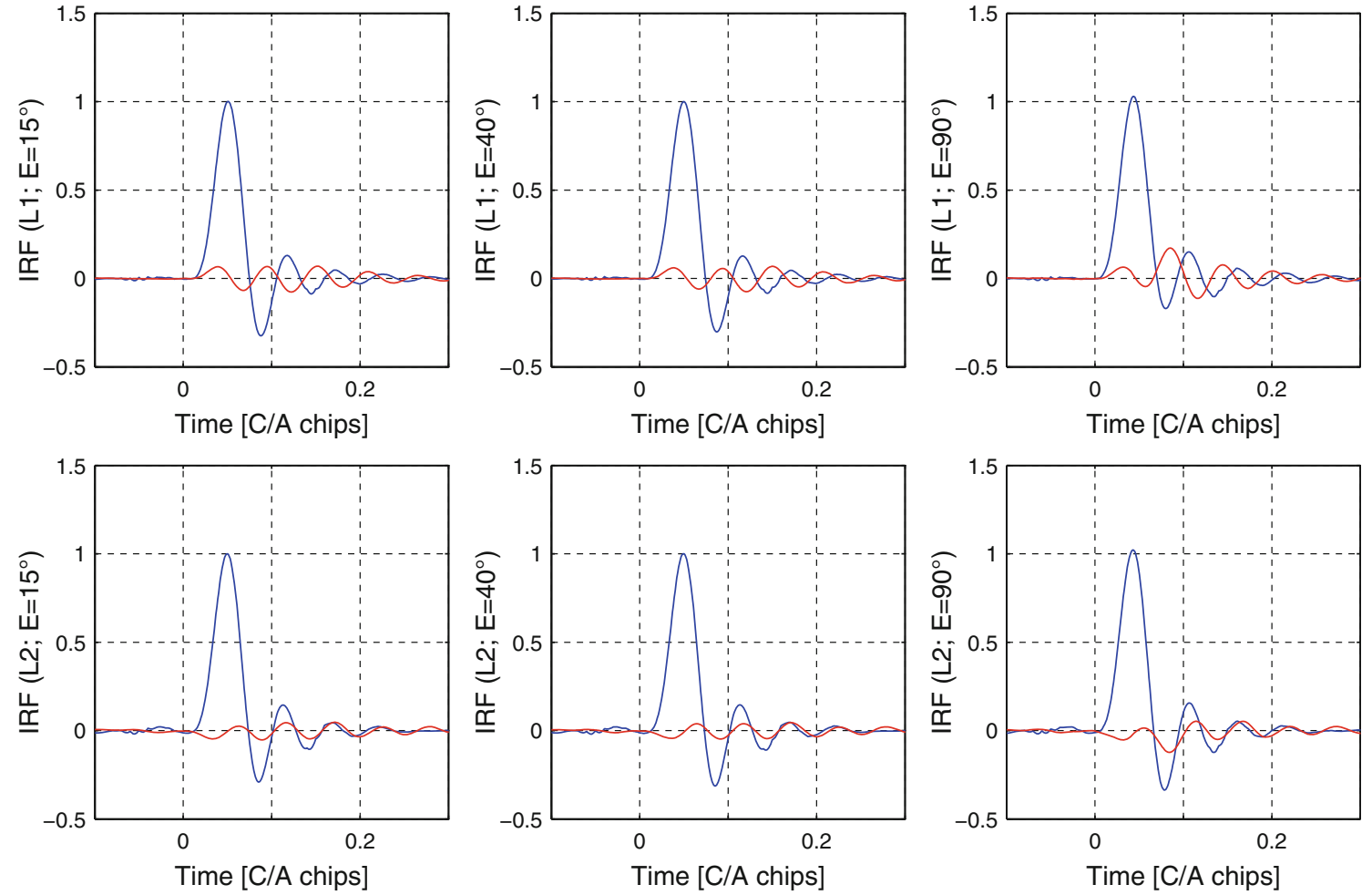

Fig. 4 Real (blue) and imaginary (red) part of the impulse response function for the SVN49 L1 and L2 signal observed at $15^{\circ}($ left $), 40^{\circ}($ center $)$ and $90^{\circ}$ (right) elevation on 16 April 2010 
For the present analysis, a different approach is pursued (Meurer et al. 2010), which does not need a reference chip but builds upon the generic impulse response function rather than individual signal chip shapes. In principle, multiple reflected signal paths may need to be considered in this step. However, for the sake of simplicity, the following presentation is restricted to a single reflection. This has been found adequate to explain the observed multipath contribution in the SVN49 signals.

The impulse response function may then be written as the sum

$\underline{h}(t)=\underline{h}_{0}(t)+\alpha(\theta) \cdot e^{+j \varphi(\theta)} \cdot \underline{h}_{0}(t-\tau(\theta))$

of the impulse response $\underline{h}_{0}(t)$ for a multipath-free transmission system with band limitation and a time and phase shifted copy thereof. Here, in accord with the notation introduced in Part 1, $\tau$ designates the total delay of the multipath channel, $\alpha$ is the relative amplitude and $\varphi$ is the associated phase shift. Both the observed amplitude and phase of the multipath component depend strongly on the boresight angle $\theta$, or equivalently the elevation $E$, due to the varying gain and phase shift introduced by the inner and outer antenna ring. On the other hand, the antenna system is considered to be free of elevation-dependent group delay variations that would otherwise have been identified in any other GPS satellite. As such, the observed delay of the reflected signal can be assumed to match the total path delay $\tau_{\mathrm{R}}$ from the $\mathrm{J} 1$ input of the coupler network to the reflection point and back to the $\mathrm{J} 2$ input (see Part 1, Fig. 1). Within the subsequent analysis, $\tau$ is adjusted as a free parameter without a priori constraints but the above assumption is well confirmed by the measured values.

For the estimation of the multipath parameters, the loss function

$J=\left\|\frac{H(f, \theta)}{H_{0}(f)}-\left(1+\underline{\alpha}(\theta) \cdot e^{-j 2 \pi f \tau(\theta)}\right)\right\|$

with $\alpha(\theta)=\alpha(\theta) \cdot e^{+j \varphi(\theta)}$ is minimized, where $H(f, \theta)$ denotes the Fourier transform of the total impulse response function, which is known from observation at frequency $f$ and boresight angle $\theta$. Likewise, $H_{0}$ is the Fourier transform of the multipath-free impulse response function, which is here treated as unknown. The joint retrieval of multipath parameters and the multipath-free IRF is generally possible, because the latter is applicable for all elevations and an optimum estimate can thus be obtained by simultaneous processing of observed IRFs over a range of different elevations.

Overall, the minimization of (6) constitutes a highly nonlinear problem, which can only be solved in a stepwise approach (Meurer et al. 2010). The estimation starts with an initial estimate $H_{0}^{(0)}$ of the multipath-free transfer function which, in case of SVN49, is obtained from the
IRF at boresight angles with a strongly suppressed gain of the $\mathrm{J} 2$ antenna input port (roughly $40^{\circ}$ for L1 and $30^{\circ}$ for L2). An estimate of the path delay $\tau$ is then obtained through a matrix pencil method (Sarkar and Pereira 1995). Subsequently, the multipath amplitude and phase shift can be adjusted, which represents a fully linear estimation problem, when formulated in terms of the real and imaginary part of the complex valued amplitude/ phase parameter $\underline{\alpha}(\theta)$. A refined estimate of the multipath-free transfer function can now be obtained by back substitution of the estimated multipath parameters into

$H_{0}(f)=\frac{H(f, \theta)}{\left(1+\underline{\alpha}(\theta) \cdot e^{-j 2 \pi f \tau(\theta)}\right)}$

and averaging over all elevations. Upon iteration, an optimal value of $H_{0}$ can thus be obtained along with the elevation-dependent multipath parameters, which is free of a priori assumption.

In order to illustrate the analysis, Fig. 1 shows the recovered multipath-free impulse response function as well as the separated multipath contribution for a sample of L1 measurements collected at high elevations. The time delay, relative power, and phase of the observed multipath contribution are provided in Fig. 5, for both L1 and L2 frequency. From the L1 measurements at high elevations, a path delay of $40.0 \pm 0.5 \mathrm{~ns}$ (roughly $12 \mathrm{~m}$ ) is obtained. Evidently, the uncertainty of the delay computation increases notably by up to a factor of ten for elevations with a strongly attenuated multipath contribution. Overall, however, the measurements at individual elevations are generally compatible with the expectation of a constant delay when taking into account the statistical uncertainties. Likewise, the L2 measurements are compatible with the above value within the associated standard deviation. For $\mathrm{L} 1$, a peak power of the reflected signal contribution of $15 \mathrm{~dB}$, which corresponds to $18 \%$ amplitude ratio, is obtained near zenith. Near $40^{\circ}$ the reflected signal power vanishes almost completely and increases back to $-32 \mathrm{~dB}$, or $2.5 \%$ amplitude ratio for low elevation observations. The phase shift of the reflected signal component relative to the direct signal varies from $+30^{\circ}$ to $-30^{\circ}$ between zenith and $50^{\circ}$ elevation. Near the antenna gain minimum, the phase is inverted and a relative phase of $-210^{\circ}$ is observed close to the horizon. In case of L2, the reflected signal power is generally much lower and confined to a peak value of $22 \mathrm{~dB}$, or equivalently $8 \%$ of the amplitude.

The estimated values for the reflected signal power compare favorably with the measurements of the relative gain of the $\mathrm{J} 1$ and $\mathrm{J} 2$ input ports of the antenna coupler network that have earlier been obtained at the compact antenna test range in Newtown, Pennsylvania (Ericson et al. 2010). Here, a null of the J2-J1, gain pattern for L1 

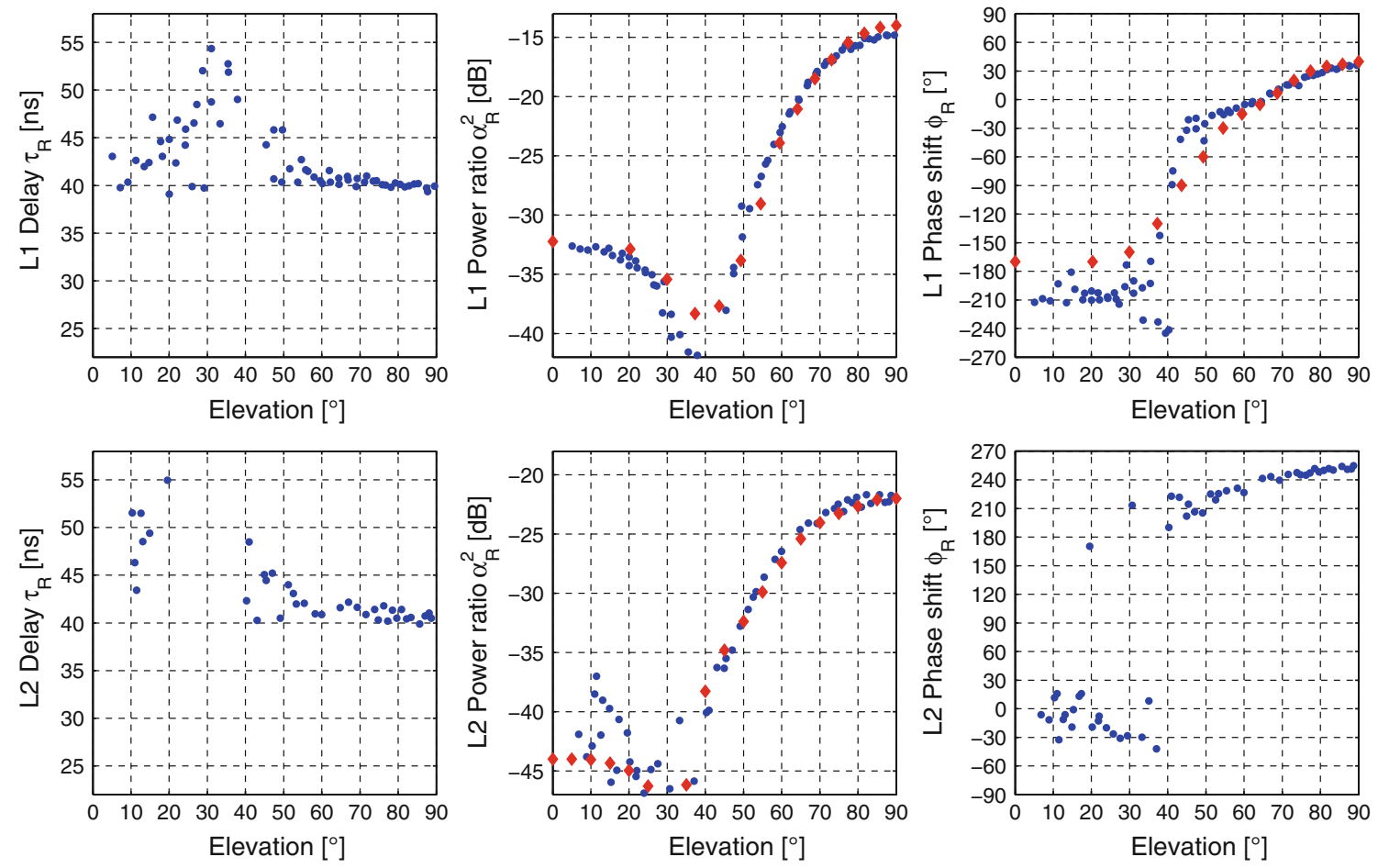

Fig. 5 Estimated multipath parameters (relative delay (left), power (center), phase (right)) for L1 (top) and L2 (bottom). Red diamonds show modeled values of the L1 power and phase shift variations based on ground-based calibrations of the Block-IIF antenna system

signals was observed at $10^{\circ}$ boresight angle, which corresponds to a $43^{\circ}$ elevation and matches the observed drop of the multipath contribution in Fig. 5. Comparing the measured J2-J1 gain near zenith $(+11 \mathrm{~dB})$ with the observed reflected signal power $(-15 \mathrm{~dB})$, it can be inferred that a $26 \mathrm{~dB}$ power fraction ( $5 \%$ amplitude) of the L1 signal fed into the $\mathrm{J} 1$ port re-enters the $\mathrm{J} 2$ port after leaking out of the coupler and being reflected at the L5 test payload. For L2, a good match of the observed multipath power with antenna gain patterns from Lake and Stansell (2009) is likewise obtained except for observations below a $30^{\circ}$ elevation.

The estimated phase offset between the direct and reflected L1 signal follows the same trend as the J2-J1 phase difference measured in the antenna test range, but shows a more rapid and steep phase inversion $\left(180^{\circ}\right.$ shift $)$ near $40^{\circ}$ elevation as well as a systematic bias at low elevations. While these differences may in part be attributed to the uncertainty of the phase delay estimation at low multipath amplitudes, independent measurements with a Vision Correlator support the correctness of the estimated values.

Compared to the analysis of the IQ histograms presented in Part 1 of this study, the chip shape analysis was found to deliver more robust and reliable estimates of the multipath parameters and provides a notable reduction in the overall computational effort. from Ericson et al. (2010). For L2 the observed multipath power is compared against antenna gain variations provided in Lake and Stansell (2009)

\section{Vision Correlator}

The Vision Correlator of the OEMV receiver (Fenton and Jones 2005) directly measures the C/A code chip shape relative to a reference time given by the tracking point of the standard early-minus-late or double-delta correlator of the receiver. Besides the in-phase channel, which is aligned with the phase of the C/A code signal, the Vision Correlator provides a quadrature channel that senses the leakage of signal power during chip transitions as well as phaseshifted multipath components.

Representative chip profiles obtained with the Vision Correlator at different elevations are shown in Fig. 6 for the zenith pass of SVN49 on April 15, 2010. The raw Vision Correlator measurements have been averaged over $1^{\circ}$ elevation bins and normalized to unit amplitude outside the transition region, where the I component changes from -1 to +1 . Small differences in the chip shape at a given elevation may be recognized between the ascending and descending branch. These might reflect azimuth dependent asymmetries in the transmit antenna design, but will require further investigation. In accord with the raw sampling results (Figs. 2, 3), a ringing may be noticed after the chip transition for observations collected at medium and low elevations. Since the bandwidth of the receiver front end $(20 \mathrm{MHz})$ is similar to the bandwidth limitation of the transmitted signal, 

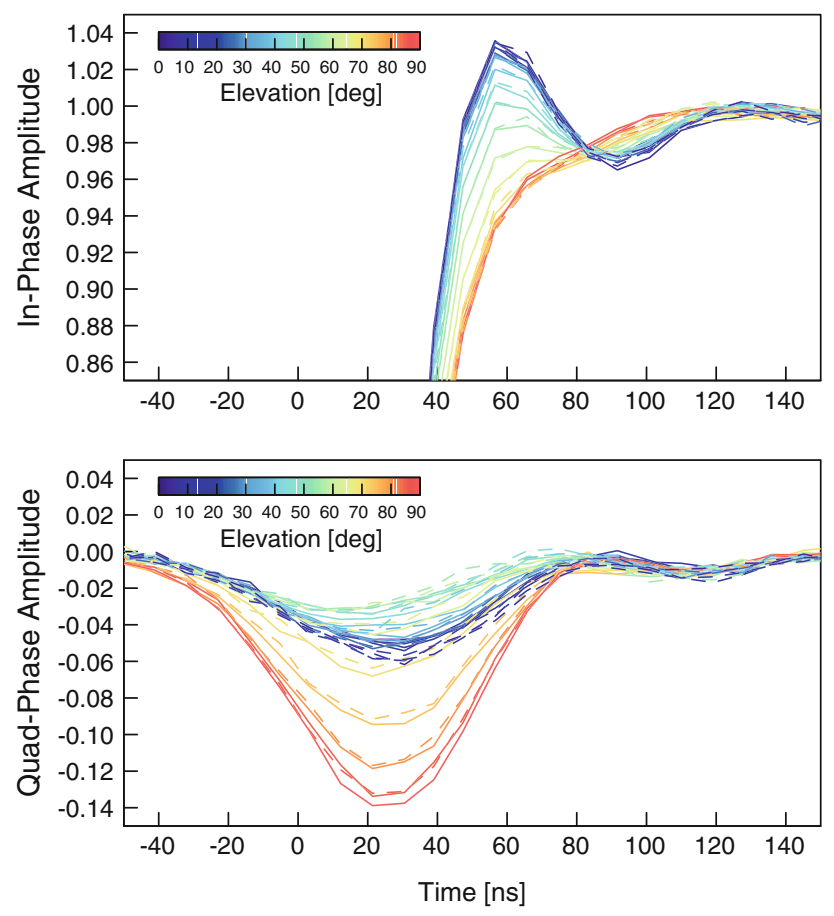

Fig. 6 Chip shapes measured with the OEMV Vision Correlator on April 15, 2010 at $5^{\circ}$ elevation steps relative to the tracking point of the standard correlator. Solid and dashed lines distinguish the ascending and descending branches of the pass

almost identical chip deformations may be observed with the Vision Correlator. At high elevations, in contrast, the chip profile is smeared out due to the pronounced contribution of the reflected signal and shows hardly any overshooting.

For maximum independence from the previous analysis, the algorithm of Weill (2002) was employed to decompose the observed Vision Correlator chip shapes into a direct and reflected component making use of the $40^{\circ}$ elevation chip shape as a reference. The results of this analysis are presented in Fig. 7 and show a remarkably good overall agreement with the raw sampling results of Fig. 5. Again, a close match of the estimated multipath amplitude with the measured antenna gain patterns is obtained, whereas some systematic differences can be observed in the estimated phase. Even though the Vision Correlator is restricted to L1 C/A code tracking, it enables a proper analysis of the satellite-induced SVN49 multipath in this frequency band and provides a notably easier access to chip shape observations than the spectrum analyzer data.

\section{Correlator synthesis}

The chip shapes derived from the raw sampling or Vision Correlator can be used to compute the response of arbitrary correlator types and thus to predict the pseudorange error caused by the SVN49 multipath. As a common example, we consider an early-minus-late (E-L) correlator with an $\mathrm{E}-\mathrm{L}$ chip spacing $d$. The $\mathrm{E}$ and $\mathrm{L}$ correlation results are obtained by forming the product of the observed signal with time-shifted copies of the ideal, rectangular, chip form and subsequent integration over the chip duration (Ward et al. 2006). When assuming a $-1 /+1$ representation of the low and high chip states, the $\mathrm{E}-\mathrm{L}$ error signal for a time $t$ of the respective prompt channel thus matches the integral of the observed chip shape between the epochs $t-d / 2$ and $t+d / 2$ relative to the nominal chip transition and the tracking point of the correlator is given by the root of the resulting function. The tracking point is separately computed for the multipath affected chip shape (including the direct and reflected signal) and the direct signal itself that has been recovered as part of the multipath estimation. The difference between the two tracking points then provides the multipath pseudorange error for the respective correlator spacing.

Results for C/A code tracking with a wide ( $d=1$ chip), narrow ( $d=0.1$ chip), and ultra-narrow ( $d=1 / 30$ chip) E-L correlator are shown in Fig. 8 (left). At high elevation, a peak multipath error of $+1.7 \mathrm{~m}$ is predicted for the wide correlator, whereas a negative error of $-0.2 \mathrm{~m}$ is obtained at low elevation. In accord with the vanishing multipath amplitude near $40^{\circ}$ elevation, the predicted pseudorange error also vanishes at this elevation. The synthesized correlator outputs for both the wide and ultra-narrow correlator closely match the actual receiver measurements presented in Part 1 of this study and thus provide a final confirmation for the applicability of a single-path reflection model. A less-good fit is obtained for the 0.1 chip narrow correlator case, though, which may indicate that the employed correlator actually has a slightly narrower effective spacing than assumed. For the case of L2 (Fig. 8, right), the synthesized pseudorange errors for the 1 chip E$\mathrm{L}$ correlator are again in good agreement with the tracking receiver results, except in the $20^{\circ}-30^{\circ}$ elevation range, where the multipath parameters from the raw R/F sampling show a high uncertainty due to the very low multipath power.

\section{Summary and conclusions}

Raw radio frequency samples collected with a high-gain antenna and derived chip shapes have been used to characterize the satellite inherent multipath of the SVN49 (PRN1) spacecraft. Compared to previous investigations, the results presented here address both the L1 and L2 frequency and apply for all signal modulations. For comparison, L1 C/A code chip shapes from a Vision Correlator receiver have been analyzed and a good match is obtained with the raw sampling results. For the derivation of 

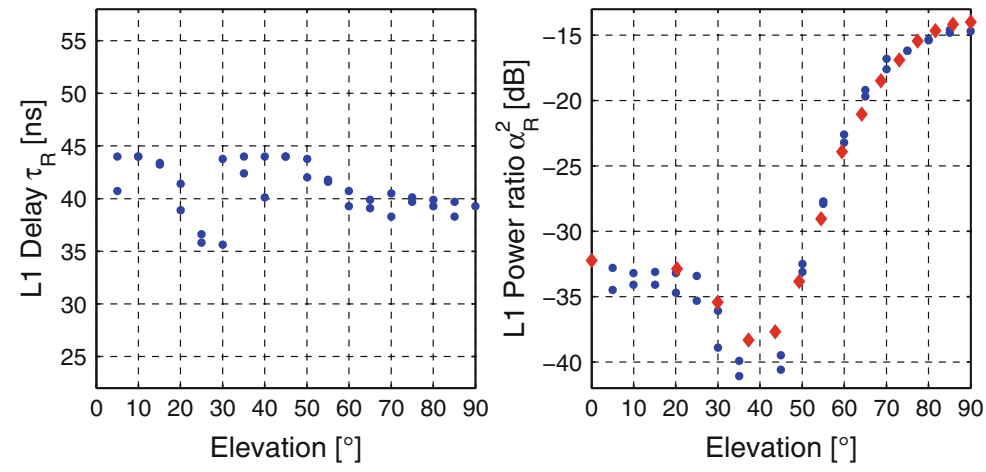

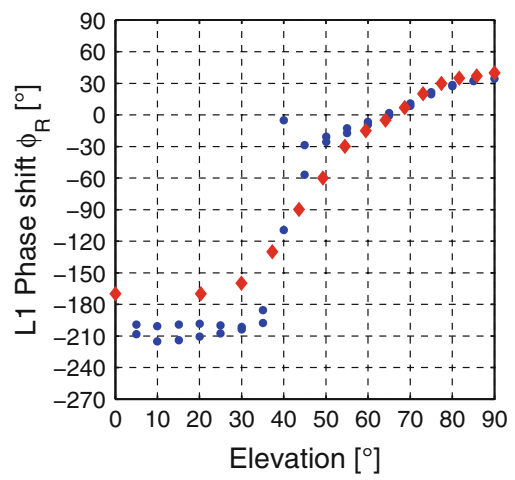

values of the L1 power and phase shift variations based on groundbased calibrations of the Block-IIF antenna system from Ericson et al. (2010)

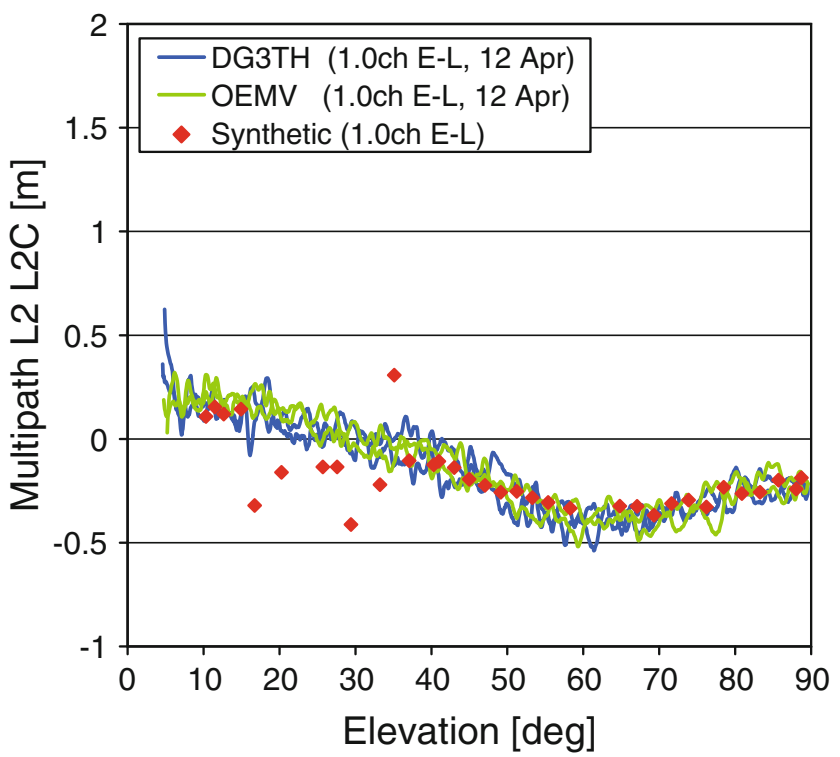

Fig. 8 Comparison of synthesized early-late correlator responses based on observed SVN49 C/A-code (left) and L2C-code chip shapes (right) with pseudorange multipath error of actual tracking receiver measurements based on standard early-minus-late correlators

multipath parameters, the characteristics of the transmission system are described in terms of the impulse response function. A newly developed method is applied to separate the multipath contributions with no restricting a priori assumptions on the multipath-free response function or chip shape. A single reflection path is found to be adequate to describe the SVN49 conditions, and synthesized multipath profiles agree well with actual receiver measurements.

Along with the results presented in Part 1 of this study, a comprehensive characterization of the SVN49 multipath is achieved, which supports the design of different forms of mitigation methods. This includes the implementation and tuning of multipath-mitigating correlators, the configuration of test scenarios in GPS signal simulators, and the derivation of reference multipath profiles for a posteriori correction of affected pseudorange measurements. Due to their generic nature, the results of this study can be used to understand the response of arbitrary receiver types and are likewise applicable for mass market, geodetic, and civil receivers.

Acknowledgments The authors would like to thank the GPS Wing, Aerospace Corporation and Stansell Consulting Inc. for fruitful discussions and technical support in setting up the SVN49 test campaign. We are also grateful for the vital support of the Weilheim operations team that has notably contributed to the successful performance of the SVN49 tracking. Receivers and test specific firmware modifications for the data collection have been provided by Javad GNSS, Navcom, and NovAtel. The support of all manufacturers is equally acknowledged. The activities have been funded within the frame of DLR's Center for Excellence for Satellite Navigation.

Open Access This article is distributed under the terms of the Creative Commons Attribution Noncommercial License which permits any noncommercial use, distribution, and reproduction in any medium, provided the original author(s) and source are credited. 


\section{References}

Ericson S, Shallberg K, Edgar C (2010) Characterization and simulation of SVN49 (PRN01) elevation dependent measurement biases. In: Proceedings of ION ITM 25-27 January 2010, San Diego, California, USA

Fenton P, Jones J (2005) The theory and performance of NovAtel Inc.'s Vision Correlator. In: Proceedings of the ION GNSS 2005, Long Beach, CA, September 2005, pp 2178-2186

Hauschild A, Montenbruck O, Erker S, Thoelert S, Meurer M, Ashjaee J (2011) A multi-technique approach for characterizing the SVN49 signal anomaly, part 1: receiver tracking and IQ constellation. GPS Solutions. doi: 10.1007/s10291-0110203-2

Lake J, Stansell T (2009) SVN-49 signal anomaly. In: Presented at: 49th meeting civil GPS service interface committee (CGSIC), Savannah, 21 September 2009

Meurer M, Thoelert S, Erker S, Montenbruck O (2010) Potentials for GNSS signal enhancements - an assessment of the impact of satellite imperfections on the navigation performance of today and future GNSS. In: Proceedings ION-GNSS-2010, 21-24 Sep 2010, Portland, Oregon

Sarkar TK, Pereira O (1995) Using the matrix pencil method to estimate the parameters of a sum of complex exponentials. IEEE Antennas Propag Mag 37(1):48-55

Thölert S, Erker S, Meuer M (2009) GNSS signal verification with a high gain antenna - calibration strategies and high quality signal assessment. ION ITM 2009, 26-28 January 2009, Anaheim, California

Ward PhW, Betz JW, Hegarty ChJ (2006) Satellite signal acquisition, tracking and data demodulation. In: Hegarty ChJ, Kaplan ED (eds) GPS—principles, applications, 2nd edn. Artech House, Boston

Weill RL (2002) Multipath mitigation using modernized GPS signals: how good can it get? In: ION GPS 2002: 15th international technical meeting of the Satellite Division of The Institute of Navigation, Portland, OR, USA, 24-27 Sept 2002

Whalen AD (1971) Detection of signals in noise. Academic Press, New York

\section{Author Biographies}

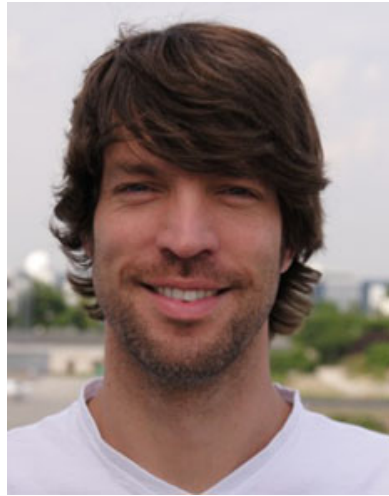

Steffen Thoelert received his diploma degree in Electrical Engineering at the University of Magdeburg, Germany in 2002. Since the same year he has been a member of the scientific staff at the German Aerospace Center (DLR). In 2006, he joined the Institute of Communications and Navigation at the German Aerospace Centre (DLR). His current work is in the fields of GNSS verification and system calibration.

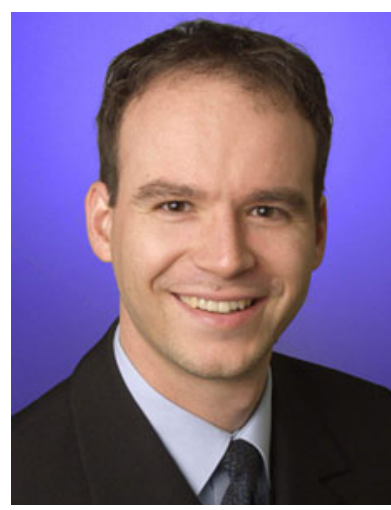

Dr. Michael Meurer is head of the Department of Navigation at DLR's Institute for Communications and Navigation, and coordinating director of the DLR Centre of Excellence for Satellite Navigation. Since 2005 he has been an Associate Professor (PD) at the Technical University of Kaiserslautern. His research interests include GNSS signals, GNSS receivers, interference mitigation and navigation for safety-critical applications.

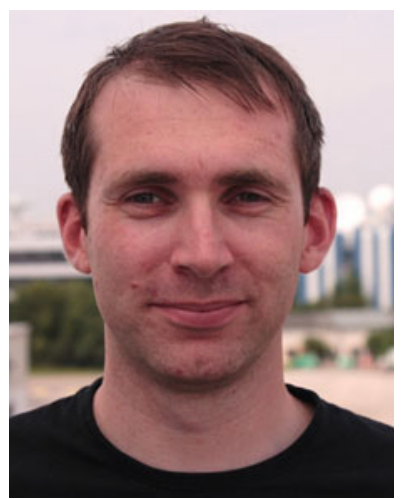

Stefan Erker received his diploma degree in Communication Technology at the Technical University of Kaiserslautern, Germany in 2007. In the same year he joined the Institute of Communications and Navigation of the German Aerospace Center (DLR) at Oberpfaffenhofen. He mainly works on the topics of GNSS verification and corresponding signal analysis.

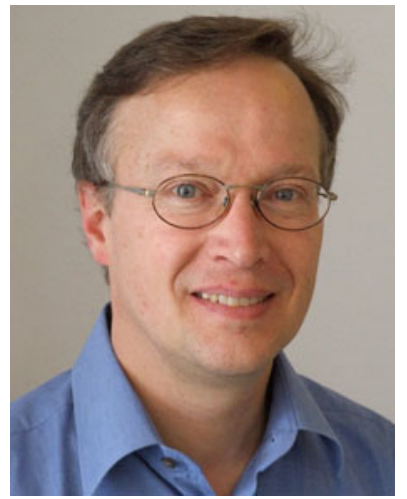

Dr. Oliver Montenbruck is head of the GNSS Technology and Navigation Group at DLR's German Space Operations Center, where he started to work as a flight dynamics analyst in 1987. His current research activities comprise spaceborne GNSS receiver technology, autonomous navigation systems, spacecraft formation flying, precise orbit determination, and multi-constellation GNSS.

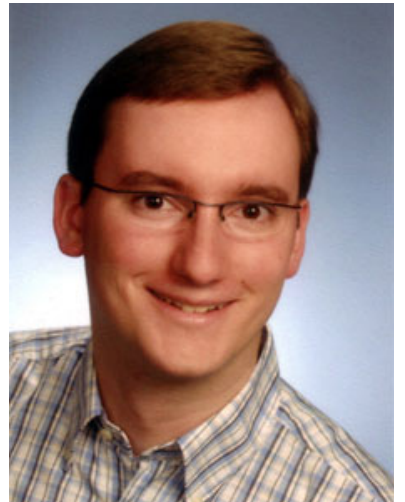

Dr. André Hauschild is a scientific staff member at DLR's German Space Operations Center (GSOC). His field of work focuses on precise real-time orbit and clock estimation for GNSS satellites as well as multi-GNSS processing using modernized GPS and new satellite navigation systems. 


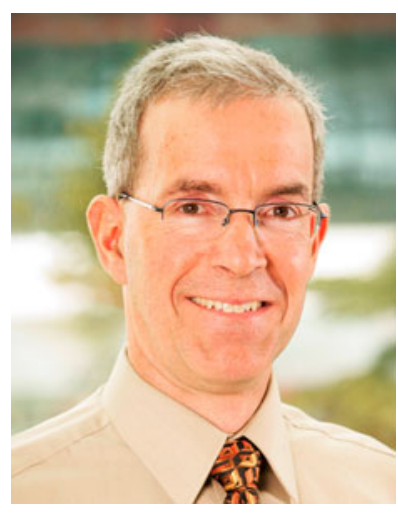

Pat Fenton received his B.Sc. in surveying Engineering from the University of Calgary in 1981. After working in the Oil and Gas exploration industry he joined NovAtel Inc. in 1989, where he led the engineering development teams through several generations of GNSS receiver products. He authored many GNSS technical papers and patents. Pat currently leads the research group exploring advanced PVT technologies and holds the position of Vice

President and Chief technology officer 